\title{
COPD exacerbation care bundle improves standard of care, length of stay, and readmission rates
}

\author{
This article was published in the following Dove Press journal: \\ International Journal of COPD \\ 17 March 2016 \\ Number of times this article has been viewed
}

\author{
Raj Parikh' \\ Trushil G Shah ${ }^{2}$ \\ Rajive Tandon ${ }^{2}$ \\ 'Department of Internal Medicine, \\ ${ }^{2}$ Department of Pulmonary and \\ Critical Care Medicine, Rush \\ University Medical Center, Chicago, \\ IL, USA
}

Introduction: COPD is the third leading cause of death in the world. Utilizing care bundles during acute COPD exacerbations results in fewer complications and lower costs. Our aim was to construct a COPD exacerbation care bundle and evaluate the effects on patient care.

Methods: We conducted a prospective analysis of 44 patients admitted with a COPD exacerbation to a single tertiary care facility. Primary outcomes included length of stay, readmission rates, and hospital costs. Secondary outcomes included patient education, pulmonologist follow-up, and timeliness of medication administration. Two cohorts were analyzed: those treated with an electronic COPD care bundle (cases; $\mathrm{N}=22$ ) versus those treated without the care bundle (controls; $\mathrm{N}=22$ ).

Results: Mean length of stay (51.2 vs 101.1 hours in controls; $P$-value $=0.001$ ), 30 -day readmission rates $(9.1 \%$ vs $54.4 \%$ in controls; $P$-value $=0.001)$, and 60 -day readmission rates $(22.7 \%$ vs $77 \%$ in controls; $P$-value $=0.0003)$ decreased in the care bundle group. Ninety-day hospital costs had a significant difference in the care bundle group (US\$7,652 vs US\$19,954 in controls; $P$-value $=0.044)$. Secondary outcomes included a $100 \%$ rate of COPD inhaler teaching (vs $27.3 \%$ in controls; $P$-value $<0.001$ ), $59.1 \%$ rate of pulmonologist follow-up after discharge (vs $18.2 \%$ in controls; $P$-value $=0.005$ ), and a mean reduction in time to steroid administration ( 7.0 hours; $P$-value $=0.015$ ) seen in the care bundle cases.

Conclusion: Our significant findings coupled with the recent success of standardized algorithms in managing COPD exacerbations stress the importance of enforcing clinical guidelines that can enhance patient care. We demonstrated improved care for COPD exacerbation patients during hospitalizations, thereby decreasing morbidity and the financial burden hospitals face in regard to this increasingly prevalent disease.

Keywords: acute exacerbation of chronic obstructive lung disease, COPD, care bundle

\section{Introduction}

COPD is a respiratory condition characterized by airflow inflammation that has become the third-ranked cause of death in the world. ${ }^{1}$ COPD is not only a leading cause of mortality and morbidity worldwide but also has translated to a significant economic and social burden that continues to expand. ${ }^{2}$ An acute COPD exacerbation is defined as a change in the patient's baseline respiratory status and development of respiratory symptoms that generally warrants an adjustment in the patient's regular medication regimen. ${ }^{3}$ COPD exacerbations are associated with increased mortality, with in-hospital mortality ranging from $3 \%$ to $9 \% .{ }^{4}$ As a consequence of high prevalence, COPD exacerbations involve significant resource utilization, including frequent clinic visits, multiple hospitalizations, and chronic therapeutic interventions. ${ }^{5}$

The burden of COPD on the health care system is directly related to the increasing number of COPD exacerbations. Global health care costs for COPD exacerbations
Correspondence: Raj Parikh

Department of Internal Medicine, Rush University Medical Center, 1653 West Congress Parkway, Chicago, IL 606I2, USA

Tel +l 8605585963

Email raj_parikh@rush.edu
International Journal of COPD 2016:1 I 577-583

(c) (i) (2) () 2016 Parikh et al. This work is published and licensed by Dove Medical Press Limited. The full terms of this license are avalable at https://www.dovepress.com/terms.php cc) hereby accept the Terms. Non-commercial uses of the work are permitted without any further permission from Dove Medical Press Limited, provided the work is properly attributed. For permision for commercial use of this work, please see paragraphs 4.2 and 5 of our Terms (https://www.dovepress. con/terms.php).

577 you
Dovepress

http://dx.doi,org/10.2147/COPD.S100401 
increased by $\sim 40 \%$ between the 1980 s and 2000 s, and since then, have continued to increase at $\sim 5 \%$ annually and are projected to increase by $60 \%$ by the year $2030 .^{5,6}$ Recent estimates suggest that aggregate costs for COPD exacerbations in US are $\sim \$ 4$ billion. ${ }^{5}$ In Europe, COPD accounts for more than half of the total health care budget attributed to all respiratory diseases, and estimates of $\$ 15$ million per 100,000 patients have been calculated. ${ }^{7,8}$

Health care costs attributed to COPD continue to escalate because of increasing length of stay and readmission rates. Length-of-stay rates for acute COPD exacerbation range from 4.5 to 8.8 days. ${ }^{9}{ }^{10}$ Meanwhile, COPD is now the fourth leading cause of 30-day readmissions. ${ }^{11}$ In fact, COPD readmissions, along with cardiac disease, cause the largest number of readmissions globally. ${ }^{12}$ Factors associated with increased readmission rates in COPD patients have been studied, and attempts to establish risk predictors have been made. ${ }^{13,14}$ In particular, variables for increased readmission rates include longer length of stay during initial hospitalization, COPD severity as defined by Global initiative for chronic Obstructive Lung Disease criteria, comorbidities, and age of patients. ${ }^{15-18}$ Subsequently, these same factors have shown to play a direct role in increasing hospital costs of COPD patients and increase health care resource utilization. ${ }^{19-22}$

Inappropriate use of medications during and after hospitalizations and failure to receive appropriate referrals for subspecialty care and pulmonary rehabilitation are key components to the substandard care being currently practiced for COPD exacerbations. An expanding body of literature states that providing clinicians with access to automated clinical decision support tools can improve quality of care and patient safety. A prime example of a clinical decision support tool is the standardized care bundle, which has become fundamental in shaping and improving patient care. The use of standardized care bundles has gained popularity in recent years with the increased utilization of electronic medical records (EMRs).

Evidence suggests that the use of automated tools during inpatient management of patients results in fewer complications, lower mortality rates, and lower costs. ${ }^{23}$ One study evaluated the use of an evidence-based care bundle in cirrhotic patients presenting with gastrointestinal bleeding. ${ }^{24}$ Factors such as timeliness of antibiotic and octreotide administration were measured, with better results seen in the experimental group that utilized the care bundle. Similarly, $>3,000$ patients were studied in an investigation examining the use of a care bundle in patients presenting with pneumonia. ${ }^{25}$ Results were significant for decreased inpatient mortality and reductions in hospital costs in the experimental arm that used the pneumonia care bundle. Multiple studies evaluating the use of standardized care bundles in the management of heart failure exacerbations were able to show reduced 30-day mortality and 30-day readmission rates. ${ }^{26-28}$ Meanwhile, evidence-based care bundles have been used in the management of sepsis and septic shock with results significant for earlier administration of antibiotics, improved 30-day mortality, less utilization of vasopressors, and decreased length of stay. ${ }^{29-31}$ Studies that examined care bundle use for surgical admissions have also proven to reduce length of stay and hospital costs. ${ }^{32-35}$

Creating an evidence-based care bundle that applies the recommended guidelines for COPD exacerbations can directly have a beneficial effect on patient care and health care costs. ${ }^{36}$ Our study's aim was to construct a COPD exacerbation care bundle and then evaluate its effects on clinical practice and outcomes.

\section{Methods}

\section{Hospital care bundle}

The COPD exacerbation care bundle was developed by a committee including critical care physicians, pulmonologists, general internists, pharmacists, respiratory therapists, and registered nurses. The content of the care bundle was driven by the recommended guidelines for COPD exacerbations. ${ }^{34}$ Given the simplified use of the care bundle, formal training was not required for incorporating the care bundle into management plans. The electronic care bundle was defined as the COPD module.

Specifically, the COPD module included sections focusing on standard nursing protocols, patient education regarding appropriate inhaler technique, and medication options for inhalers, nebulizers, steroids, and antibiotics (Figure 1). An additional aspect of the order set focused on important measures at time of discharge, including providing patients with referrals to see outpatient pulmonologists (Figure 2). The COPD module was incorporated into the hospital's medical record system, Epic (Epic, Verona, WI, USA; 2010). The decision to use the COPD module and its components was at the discretion of the treating physician, and the investigators had no influence on whether or not the treating physicians opted to use it.

\section{Study design}

A prospective, observational study design was used, enrolling consecutive patients who were admitted to Rush University Medical Center from January 2015 to June 2015 with COPD exacerbations, as defined by their International Classification of Diseases-9 coding. The Institutional Review Board of 


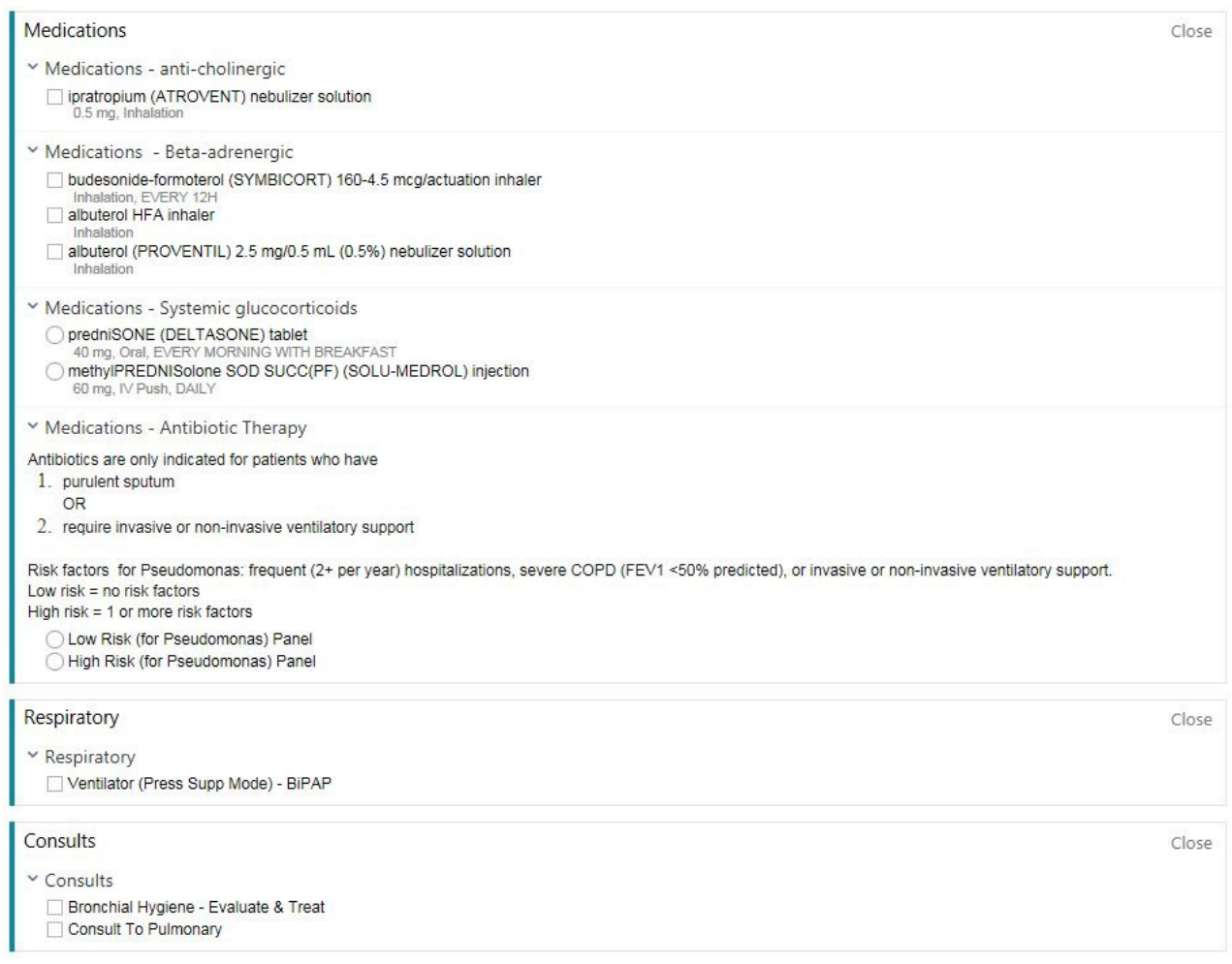

Figure I Medication options for inhalers, nebulizers, steroids, and antibiotics.

Rush University Medical Center approved the study, and the requirement for informed consent was waived as use of the care bundle was the decision of the ordering physician (ORA: 14092607-IRB01).

Inclusion criteria included patients admitted with COPD exacerbations. Patients admitted for varying pulmonary obstructive diseases, such as asthma and bronchitis, were excluded. Primary outcomes that were evaluated included hospital length of stay, 30- and 60-day readmission rates, and hospital costs. Secondary outcomes included patient education provided by respiratory therapists, pulmonologist follow-up after discharge, time to antibiotic administration, time to steroid administration, and time to nebulizer administration.

\section{Statistical analysis}

Mean differences in continuous variables between cases and controls were determined by independent Student's $t$-test.
Differences in nominal variables between cases and controls were determined by chi-square test. Odds ratios (ORs) were calculated for difference between 30-day readmission rates, 60-day readmission rates, length of stay, COPD inhaler teaching, time to medication administration, and pulmonologist follow-up between the groups. Mean decrease in length of stay for cases as compared to controls was calculated. All data analysis was performed using SPSS statistical software.

\section{Results}

A total of 44 patients with COPD exacerbations were analyzed. Cases were considered those who integrated the use of the COPD module ( $\mathrm{N}=22)$, and controls were those without the use of the COPD module $(\mathrm{N}=22)$. Baseline characteristics were compared between cases and controls (Table 1). The total COPD cohort population had an increased proportion that was male (59.1\%) and African

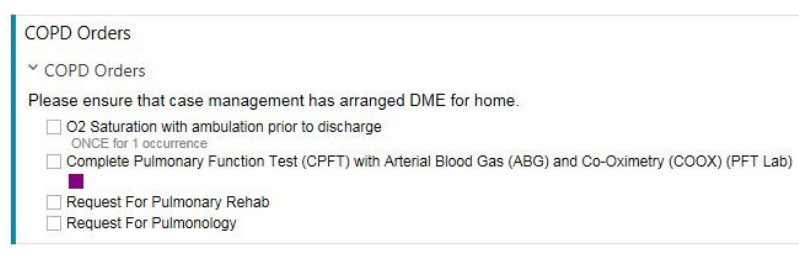

Figure 2 Orders at time of discharge. 
Table I Baseline characteristics of COPD patients admitted with exacerbation

\begin{tabular}{|c|c|c|c|c|}
\hline Characteristics & All patients $(\mathrm{N}=44)$ & Cases $(\mathbf{N}=\mathbf{2 2})$ & Controls $(\mathbf{N}=\mathbf{2 2})$ & $P$-value \\
\hline Sex & & & & 1.000 \\
\hline Male & $26(59.1 \%)$ & $13(59.1 \%)$ & $13(59.1 \%)$ & \\
\hline Female & $18(40.9 \%)$ & $9(40.9 \%)$ & $9(40.9 \%)$ & \\
\hline Age (mean) & $66.00(61.8,70.1)^{\mathrm{a}}$ & $63.45(57.3,69.6)^{\mathrm{a}}$ & $68.55(62.6,74.4)^{\mathrm{a}}$ & 0.221 \\
\hline Ethnicity & & & & 0.489 \\
\hline African American & $33(75 \%)$ & $17(77.3 \%)$ & $16(72.7 \%)$ & \\
\hline Caucasian & $10(22.7 \%)$ & $4(18.2 \%)$ & $6(27.2 \%)$ & \\
\hline Hispanic & $\mathrm{I}(2.3 \%)$ & $\mathrm{I}(4.5 \%)$ & $0(0.0 \%)$ & \\
\hline Previous PFTs & $20(44.5 \%)$ & $15(68.2 \%)$ & $5(22.7 \%)$ & 0.002 \\
\hline $\mathrm{FEV}_{1}$ (mean) & $50.2(38.7,61.3)^{\mathrm{a}}$ & $53.9(39.3,68.4)^{\mathrm{a}}$ & $41.0(18.7,63.3)^{\mathrm{a}}$ & 0.301 \\
\hline Previous COPD exacerbation hospitalizations & $32(72.7 \%)$ & $16(72.7 \%)$ & $16(72.7 \%)$ & 1.000 \\
\hline Previous appointments with pulmonologist & 18 (40.9\%) & 9 (40.9\%) & $9(40.9 \%)$ & 1.000 \\
\hline Admission to ICU & $14(3 \mathrm{I} .8 \%)$ & $8(36.4 \%)$ & $6(27.2 \%)$ & 0.322 \\
\hline Smoking status & & & & 0.680 \\
\hline Never smoker & 7 (I5.9\%) & $3(13.6 \%)$ & $4(18 \%)$ & \\
\hline Prior or current smoker & $37(84.1 \%)$ & $19(86.3 \%)$ & 18 (82\%) & \\
\hline
\end{tabular}

Note: ${ }^{\text {a } 95 \% ~ c o n f i d e n c e ~ i n t e r v a l s . ~}$

Abbreviations: PFTs, pulmonary function tests; FEV , forced expiratory volume in I second; ICU, intensive care unit.

American (75\%). Sex distribution $(P$-value $=1.000)$, mean age $(P$-value $=0.221)$, ethnicity $(P$-value $=0.489)$, and smoking status $(P$-value $=0.680)$ were similar between both cases and controls of the study.

Additional characteristics were obtained regarding both groups of patients, including severity of COPD as defined by forced expiratory volume in 1 second $\left(\mathrm{FEV}_{1}\right)$ levels. Both groups had comparable mean $\mathrm{FEV}_{1}$ (cases: $53.9 \%$, controls: $41.0 \% ; P$-value $=0.301)$, comparable percentage of prior admissions for COPD exacerbations (cases: $72.7 \%$, controls: $72.7 \%$; $P$-value $=1.000$ ), and a comparable proportion of prior appointments with pulmonologists (cases: $40.9 \%$, controls: $40.9 \%$; $P$-value $=1.000$ ). In total, 14 patients $(31.8 \%)$ were admitted to the intensive care unit, eight of those being cases (36.4\%) and six being controls $(27.2 \%)$.

Mean length of stay (in hours), 30- and 60-day readmissions, and 90-day aggregate hospital costs were assessed as primary outcomes (Table 2 ). The experimental group had a mean length of stay of 51.2 hours $(\sim 2.13$ days). The control group had a mean of 101.1 hours ( 4.21 days). A mean difference of 49.95 hours $(P$-value $=0.001)$ suggests a significant reduction in length of stay with use of the COPD module. Meanwhile, two patients $(9.1 \%)$ had a 30 -day readmission in the experimental group, while 12 patients (54.5\%) had 30-day readmissions in the control group. For 60-day readmissions, there were five patients $(22.7 \%)$ with a readmission in the experimental group and 17 patients $(77 \%)$ in the control group.

Table 2 Differences between primary and secondary outcomes in cases and controls

\begin{tabular}{|c|c|c|c|c|}
\hline Measurable & Cases $(\mathbf{N}=\mathbf{2 2})$ & Controls $(\mathbf{N}=\mathbf{2 2})$ & $\begin{array}{l}\text { Odds ratio/ } \\
\text { mean decrease }\end{array}$ & $P$-value \\
\hline Length of stay (mean) (hours) & $51.2(34.7,67.6)^{\mathrm{a}}$ & $101.1(78.1,126.2)^{\mathrm{a}}$ & $49.95^{\mathrm{b}}$ & 0.001 \\
\hline 30-day readmission & $2(9.1 \%)$ & $12(54.5 \%)$ & $0.08^{c}$ & 0.001 \\
\hline 60-day readmission & $5(22.7 \%)$ & $17(77 \%)$ & $0.09^{c}$ & 0.0003 \\
\hline $\begin{array}{l}\text { Aggregate 90-day hospital costs } \\
\text { (mean) (USD) }\end{array}$ & $7,652(3,982, \mid I, 321)^{a}$ & $19,954(8,463,31,445)^{a}$ & $12,302^{\mathrm{b}}$ & 0.044 \\
\hline $\begin{array}{l}\text { COPD inhaler teaching provided } \\
\text { by respiratory therapist }\end{array}$ & $22(100.0 \%)$ & $6(27.3 \%)$ & $3.66^{\mathrm{c}}$ & $<0.001$ \\
\hline $\begin{array}{l}\text { Pulmonary outpatient follow-up } \\
\text { after discharge }\end{array}$ & $13(59.1 \%)$ & $4(18.2 \%)$ & $6.5^{c}$ & 0.005 \\
\hline $\begin{array}{l}\text { Time to steroid administration } \\
\text { (mean) (hours) }\end{array}$ & $2.0(1.14,2.95)^{\mathrm{a}}$ & $9.09(3.5,14.6)^{\mathrm{a}}$ & $7.0^{\mathrm{b}}$ & 0.015 \\
\hline $\begin{array}{l}\text { Time to antibiotic } \\
\text { administration (mean) (hours) }\end{array}$ & $4.3(2.6,5.9)^{\mathrm{a}}$ & $9.01(4.1,13.9)^{\mathrm{a}}$ & $4.7^{\mathrm{b}}$ & 0.069 \\
\hline $\begin{array}{l}\text { Time to nebulizer administration } \\
\text { (mean) (hours) }\end{array}$ & $3.05(1.9,4.2)^{\mathrm{a}}$ & $5.18(1.4,8.9)^{\mathrm{a}}$ & $2.12^{\mathrm{b}}$ & 0.585 \\
\hline
\end{tabular}

Notes: ${ }^{a} 95 \%$ confidence intervals. ${ }^{b}$ Mean decrease between cases and controls. ${ }^{c}$ Odds ratio between cases and controls. 
These findings support significant reduction in 30- and 60-day readmissions with use of the COPD module $(P$-value $=0.001$ and 0.0003, respectively). Difference in 90-day hospital costs ( $\$ 7,652$ in cases vs $\$ 19,954$ in controls; $P$-value $=0.044)$ was statistically significant as well. Secondary outcomes that were evaluated included a $100 \%$ rate of COPD inhaler teaching from the cases and only $27.3 \%$ in the control group $(P$-value $<0.001)$. In addition, 13 patients $(59.1 \%)$ established follow-up with a pulmonologist after discharge in the experimental arm, while only four patients (18.2\%) did from the control arm $(P$-value $=0.005)$. The COPD module group had a mean time to steroid administration of 2.0 hours $(95 \%$ confidence Interval [CI]: 1.14, 2.95), time to antibiotic administration of 4.3 hours (95\% CI: $2.6,5.9)$, and time to nebulizer administration of 3.05 hours $(95 \%$ CI: $1.9,4.2)$ as compared to 9.09 (95\% CI: 3.5, 14.6), 9.01 (95\% CI: 4.1, 13.9), and 5.18 hours (95\% CI: 1.4, 8.9), respectively, for the control group. There was a mean reduction in steroid administration by 7.0 hours $(P$-value $=0.015)$ with the use of the COPD module. There was no statistically significant reduction in time to antibiotic and nebulizer administration, however.

\section{Discussion}

\section{Interpretation of the results}

Our study shows that use of the standardized COPD module for patients admitted with COPD exacerbations reduces the length of stay by 49.95 hours (2.08 days) and significantly reduces 30- and 60-day readmission rates as well. With decreased readmissions and decreased length of stay, 90-day aggregate costs were also significantly lower in the COPD module-used group, with a mean decrease of $\$ 12,302$. These results support the use of a standardized COPD module to decrease length of stay, readmission rates, and hospital costs. In addition, we evaluated factors in our COPD module, which could have contributed to this difference. We found a statistically significant difference in COPD inhaler teaching by respiratory therapists $(\mathrm{OR}=3.66)$ and pulmonologist follow-up after discharge $(\mathrm{OR}=6.50)$. Increase in both COPD inhaler teaching by respiratory therapists and post-discharge pulmonary follow-up are the likely drivers for the decreased readmission rates. The COPD inhaler teaching by the respiratory therapists allowed patients to correctly use their inhalers, and hence, avoid a future readmission for worsening COPD. Timely post-discharge pulmonary follow-up, meanwhile, likely allowed for appropriate medication adjustments to avoid readmission as well.

For secondary outcomes, we also evaluated time to antibiotic administration, time to steroid administration, and time to nebulizer administration as surrogates for timely delivery of appropriate treatment for COPD exacerbations. We found a significant reduction in time to steroid administration by 7.0 hours $(P$-value $=0.015)$ with use of the standardized COPD module. We observed a trend in both time to antibiotic administration (mean decrease of 4.7 hours) and time to nebulizer administration (mean decrease of 2.12 hours) in the cases group, as well. These findings suggest that earlier administration of steroid and antibiotics, in addition to nebulizer therapy, resulted in improved control of COPD exacerbation symptoms, and eventually, facilitated earlier discharge. Overall, use of the standardized COPD module enabled a more efficient and timely delivery of treatment in patients with COPD exacerbations and thereby resulted in a 49.95-hour decrease in length of stay. Our data strongly suggest the use of standardized care bundles to facilitate efficient and effective treatment to patients with COPD exacerbations.

\section{Comparison to literature}

While investigations have evaluated the use of nonelectronic algorithms to standardize COPD management, an EMRbased care bundle has yet to be studied. A paper-based clinical guide for COPD management was able to show a decrease in 30 -day readmission rates from $28 \%$ to $6.25 \% .{ }^{37}$ This guide focused on importance of patient education, medication reconciliation, and post-discharge follow-up. Another similar intervention showed a reduction in hospitalincurred costs per COPD patient, decreased from $\$ 613$ to $\$ 128$ per patient. ${ }^{38}$ Care bundles, which focus on evidencebased management of COPD, have also been able to show reduced 30-day readmission rates as well as improve compliance in COPD patients. ${ }^{39}$

The significant data of secondary outcomes from our care bundle intervention have been mirrored in COPD research as well. A recent investigation showed that ensuring pulmonologist follow-up after discharge for COPD patients helps decrease readmission rates. ${ }^{40}$ Inhaler compliance and inhaler technique teaching has also been shown to decrease both 30- and 60-day readmission rates. Additionally, timeliness of appropriate COPD treatment, which was defined as within 4 hours of hospital admission, was associated with a $40 \%$ lower inpatient mortality. ${ }^{41}$

\section{Limitations}

Our study has some limitations. Firstly, it was performed within a single tertiary care institution in a university hospital setting, which may not be applicable to other 
treatment settings. Secondly, the possibility of selection bias exists where potentially stable patients received the COPD module more often than acutely ill patients. However, our baseline characteristics of mean $\mathrm{FEV}_{1}$, mean age, and similar rates of admissions to the intensive care unit show that both groups were relatively similar in regard to severity of illness. Regardless, the decision of whether or not to use the COPD module was left to the treating physician. Lastly, our population number was not powered to reflect mortality benefit. In the future, a multicenter and long-term investigation is warranted in order to evaluate a larger patient population and further distinguish the potential impact of a care bundle, as EMRs are becoming a more integrated and vital aspect of hospital care.

\section{Conclusion}

COPD is a disease that presents a public health challenge worldwide. Increasing mortality rates, health care costs, and prevalence are directly influencing the global health burden of the disease. This burden continues to increase as a result of continued exposure to COPD risk factors and the aging of the population. ${ }^{42}$ Given the significant findings seen in our investigation and the recent success of standardized algorithms in managing COPD exacerbation patients, the importance of establishing clinical tools that can enhance patient care cannot be understated.

Measurable outcomes from this investigation have proven the magnitude of standardized care bundles in the appropriate setting. These demonstrated results, combined with the changing health care environment and integration of EMRs, have given care bundles a new place of prominence in the hospital setting.

COPD is a disease that is directly contributing to the global health crisis by occupying significant resources, creating debilitating symptoms for patients, and worsening overall quality of life. Our study's COPD exacerbation module provides a process for translation of evidence-based therapy into everyday practice within the hospital, and by doing so, decreases the patients' morbidity and the financial burden hospitals face with this increasingly prevalent disease.

\section{Disclosure}

The authors have no relevant financial or nonfinancial relationship in regard to the information described, reviewed, and evaluated in this manuscript.

\section{References}

1. Hoyert DL, Xu JQ. Deaths: preliminary data for 2011. Natl Vital Stat Rep. 2012;61(6):1-65.
2. Mathers CD, Loncar D. Projections of global mortality and burden of disease from 2002 to 2030. PLoS Med. 2006;3:e442.

3. Rodriguez-Roisin R. Toward a consensus definition for COPD exacerbations. Chest. 2000;117(2):398S-401S.

4. Matkovic Z, Huerta A, Soler N, et al. Predictors of adverse outcome in patients hospitalised for exacerbation of chronic obstructive pulmonary disease. Respiration. 2012;84(1):17-26.

5. Blanchette CM, Gross NJ, Altman P. Rising costs of COPD and the potential for maintenance therapy to slow the trend. Am Health Drug Benefits. 2014;7(2):98-106.

6. Herse F, Kiljander T, Lehtimäki L. Annual costs of chronic obstructive pulmonary disease in Finland during 1996-2006 and a prediction model for 2007-2030. NPJ Prim Care Respir Med. 2015;26(25):15015.

7. Jansson SA, Backman H, Stenling A, Lindberg A, Ronmark E, Lundback B. Health economic costs of COPD in Sweden by disease severity - has it changed during a ten years period? Respir Med. 2013; 107:1931-1938.

8. Centers for Medicare and Medicaid Services (CMS), HHS. Medicare program; hospital inpatient prospective payment systems for acute care hospitals and the long-term care hospital prospective payment system and FY 2012 rates; hospitals' FTE resident caps for graduate medical education payment. Final rules. Fed Regist. 2011; 76(160):51476-51846.

9. Dalal AA, Shah M, D'Souza AO, Rane P. Costs of COPD exacerbations in the emergency department and inpatient setting. Respir Med. 2011; 105(3):454-460.

10. Lima FV, Yen TY, Patel JK. Trends in in-hospital outcomes among adults hospitalized with exacerbation of chronic obstructive pulmonary disease. COPD. 2015;12(6):636-642.

11. Jencks SF, Williams MV, Coleman EA. Rehospitalizations among patients in the Medicare fee-for-service program. N Engl J Med. 2009; 360(14):1418-1428.

12. Westert GP, Lagoe RJ, Keskimaki I, Leyland A, Murphy M. An international study of hospital readmissions and related utilization in Europe and the USA. Health Policy. 2002;61:269-278.

13. Yu TC, Zhou H, Suh K, Arcona S. Assessing the importance of predictors in unplanned hospital readmissions for chronic obstructive pulmonary disease. Clinicoecon Outcomes Res. 2015;7:37-51.

14. Baker CL, Zou KH, Su J. Risk assessment of readmissions following an initial COPD-related hospitalization. Int J Chron Obstruct Pulmon Dis. 2013;8:551-559.

15. Liu D, Peng SH, Zhang J, Bai SH, Liu HX, Qu JM. Prediction of short term re-exacerbation in patients with acute exacerbation of chronic obstructive pulmonary disease. Int J Chron Obstruct Pulmon Dis. 2015; 2(10):1265-1273.

16. Glaser JB, El-Haddad H. Exploring novel Medicare readmission risk variables in chronic obstructive pulmonary disease patients at high risk of readmission within 30 days of hospital discharge. Ann Am Thorac Soc. 2015;12(9):1288-1293.

17. Candrilli SD, Dhamane AD, Meyers JL, Kaila S. Factors associated with inpatient readmission among managed care enrollees with COPD. Hosp Pract (1995). 2015;43(4):199-207.

18. GOLD. Global strategy for diagnosis, management, and prevention of COPD. From the Global Initiative for Chronic Obstructive Lung Disease (GOLD). 2015. http://www.goldcopd.org. Accessed October 1, 2015.

19. Mittmann N, Kuramoto L, Seung SJ, Haddon JM, Bradley-Kennedy C, FitzGerald JM. The cost of moderate and severe COPD exacerbations to the Canadian healthcare system. Respir Med. 2008;102: 413-421.

20. Johnston NW, McIvor A, Lambert K, et al. The Christmas season as a risk factor for chronic obstructive pulmonary disease exacerbations. Can Respir J. 2010;17:275-281.

21. Mancini GB, Etminan M, Zhang B, Levesque LE, FitzGerald JM, Brophy JM. Reduction of morbidity and mortality by statins, angiotensin-converting enzyme inhibitors, and angiotensin receptor blockers in patients with chronic obstructive pulmonary disease. $J \mathrm{Am}$ Coll Cardiol. 2006;47:2554-2560. 
22. Guarascio AJ, Ray SM, Finch CK, Self TH. The clinical and economic burden of chronic obstructive pulmonary disease in the USA. Clinicoecon Outcomes Res. 2013;5:235-245.

23. Amarasingham R, Plantinga L, Diener-West M, Gaskin DJ, Powe NR. Clinical information technologies and inpatient outcomes: a multiple hospital study. Arch Intern Med. 2009;169(2):108-114.

24. Mayorga CA, Rockey DC. Clinical utility of a standardized electronic order set for the management of acute upper gastrointestinal hemorrhage in patients with cirrhosis. Clin Gastroenterol Hepatol. 2013; 11(10):1342-1348.

25. Ballard DJ, Ogola G, Fleming NS, et al. The impact of standardized order sets on quality and financial outcomes. In: Henriksen K, Battles JB, Keyes MA, et al, editors. Advances in Patient Safety: New Directions and Alternative Approaches (Vol. 2: Culture and Redesign). Rockville, MD: Agency for Healthcare Research and Quality (US); 2008.

26. Ballard DJ, Ogola G, Fleming NS, et al. Impact of a standardized heart failure order set on mortality, readmission, and quality and costs of care. Int J Qual Health Care. 2010;22(6):437-444.

27. Meleskie J, Eby D. Adaptation and implementation of standardized order sets in a network of multi-hospital corporations in rural Ontario. Healthc Q. 2009;12(1):78-83.

28. Reingold S, Kulstad E. Impact of human factor design on the use of order sets in the treatment of congestive heart failure. Acad Emerg Med. 2007;14(11):1097-1105.

29. Micek S, Roubinian N, Heauring T, et al. Before-after study of a standardized hospital order set for the management of septic shock. Crit Care Med. 2006;34(11):2707-2713.

30. O'Connor C, DeCaire K, Friedrich J. Improving patient care through the use of evidence based order sets. AMIA Annu Symp Proc. 2005:1063.

31. Ozdas A, Speroff T, Waitman LR, Ozbolt J, Butler J, Miller RA. Integrating 'best of care' protocols into clinicians' workflow via care provider order entry: impact on quality-of-care indicators for acute myocardial infarction. J Am Med Inform Assoc. 2006;13(2):188-196.
32. Santolin CJ, Boyer LS. Change of care for patients with acute myocardial infarctions through algorithm and standardized physician order sets. Crit Pathw Cardiol. 2004;3(2):79-82.

33. Takegami K, Kawaguchi Y, Nakayama H, Kubota Y, Nagawa H. Impact of a clinical pathway and standardization of treatment for acute appendicitis. Surg Today. 2003;33(5):336-341.

34. Kiyama T, Tajiri T, Yoshiyuki T, et al. Clinical significance of a standardized clinical pathway in gastrectomy patients. J Nippon Med Sch. 2003;70(3):263-269.

35. Panella M, Marchisio S, Di Stanislao F. Reducing clinical variations with clinical pathways: do pathways work? Int J Qual Health Care. 2003; 15(6):509-521.

36. Criner GJ, Bourbeau J, Diekemper RL, et al. Prevention of acute exacerbations of COPD: American College of Chest Physicians and Canadian Thoracic Society Guideline. Chest. 2015;147(4):894-942.

37. Dunne PJ. A new imperative for respiratory therapists in COPD care. Presented at: Hoag Annual Respiratory Care Conference; October 2012; New Port Beach, CA.

38. Brown GL, Dunne PJ. COPD in-patient care: time for a new paradigm. AARC Times. 2011;35(11):34.

39. Hopkinson NS, Englebretsen C, Cooley N, et al. Designing and implementing a COPD discharge care bundle. Thorax. 2012;67:90-92.

40. Shah T, Churpek MM, Coca Perraillon M, Konetzka RT. Understanding why patients with COPD get readmitted: a large national study to delineate the Medicare population for the readmissions penalty expansion. Chest. 2015;147(5):1219-1226.

41. Turner AM, Lim WS, Rodrigo C, Welham SA, Calvert JM. A carebundles approach to improving standard of care in AECOPD admissions: results of a national project. Thorax. 2015;70(10):992.

42. Lopez AD, Shibuya K, Rao C, et al. Chronic obstructive pulmonary disease: current burden and future projections. Eur Respir J. 2006; 27(2):397-412.
International Journal of COPD

\section{Publish your work in this journal}

The International Journal of COPD is an international, peer-reviewed journal of therapeutics and pharmacology focusing on concise rapid reporting of clinical studies and reviews in COPD. Special focus is given to the pathophysiological processes underlying the disease, intervention programs, patient focused education, and self management protocols.

\section{Dovepress}

This journal is indexed on PubMed Central, MedLine and CAS. The manuscript management system is completely online and includes a very quick and fair peer-review system, which is all easy to use. Visit http://www.dovepress.com/testimonials.php to read real quotes from published authors. 